\title{
Abordagem preventiva e terapêutica do distúrbio mineral e ósseo nos estágios iniciais da doença renal crônica: revisão sistemática
}

\author{
Preventive and therapeutic approach of mineral and bone disorder in the initial stages of \\ chronic kidney disease: systematic review
}

Enfoque preventivo y terapéutico del trastorno minero y hueso en las etapas iniciales de la enfermedad renal crónica: revisión sistemática

Karla Amaral Nogueira Quadros ${ }^{1 *}$, Jéssica Azevedo de Aquino ${ }^{1}$, Francisco Edson Coelho de Vasconcelos $^{2}$, João Victor Marques Guedes ${ }^{1}$, Flávio Augusto de Morais ${ }^{1}$, Fernanda Henriques Rocha Ribeiro ${ }^{1}$, Fernanda Marcelino de Rezende e Silva ${ }^{1}$, Yoshimi José Ávila Watanabe ${ }^{1}$, Alba Otoni ${ }^{1}$.

\section{RESUMO}

Objetivo: Identificar proposta padrão ouro de prevenção e tratamento para o distúrbio mineral e ósseo em adultos e idosos com doença renal crônica não-dialítica. Métodos: revisão sistemática de ensaios clínicos randomizados. Resultados: incluídos oito artigos: calcitriol ou colecalciferol, tendo calcitriol resultado melhor com correção da hipocalcemia, redução das fosfatases alcalinas séricas e do hormônio paratireóideo; alfacalcidol com controle da doença óssea em $42 \%$ dos pacientes; colecalciferol aumentou calcidiol sérico e diminuiu níveis séricos de paratormônio; colecalciferol por tempo prolongado com melhora na deficiência de vitamina $\mathrm{D}$ e diminuição dos níveis séricos de paratormônio; alendronato, não alterou a progressão da calcificação vascular; acetato de cálcio, carbonato de lantânio ou carbonato de sevelamer diminuíram o fósforo médio de urina e atenuaram a progressão do hiperparatireoidismo; calcitriol associado a intervenção dietética de cálcio, calcitriol isolado diminuiu os níveis PTHi em jejum e, quando combinado com cálcio na dieta, normalizou as respostas pós-prandiais de calcemia e paratireoide e carbonato de cálcio ou sevelamer associados a dieta restrita de fósforo. Sevelamer apresentou melhores resultados com redução significativa na mortalidade por todas as causas. Considerações finais: Não houve consenso entre os autores quanto à terapêutica padrão ouro para portadores de DMO-DRC em estágios precoces.

Palavras-chave: Distúrbio mineral e ósseo na doença renal crônica, Insuficiência renal crônica, Hiperparatireoidismo secundário.

\begin{abstract}
Objective: To identify the gold standard proposal for prevention and treatment for mineral and bone disorders in adults and the elderly with chronic kidney disease non dialysis. Methods: a systematic review of randomized controlled trials. Results: eight articles: calcitriol or cholecalciferol, with calcitriol having a better result in correcting hypocalcaemia, reducing serum alkaline phosphatases and parathyroid hormone; alfacalcidol with bone disease control in $42 \%$ of patients; cholecalciferol increased serum calcidiol and decreased serum levels of parathormone; cholecalciferol for a prolonged period with improvement in vitamin D deficiency and decreased serum levels of parathormone; alendronate for a long time did not alter the progression of vascular calcification; calcium acetate, lanthanum carbonate or sevelamer carbonate decreased the average urine phosphorus and attenuated the progression of hyperparathyroidism; calcitriol associated with dietary calcium intervention, isolated calcitriol decreased fasting PTHi levels and, when combined with dietary calcium, normalized the postprandial responses of calcemia and parathyroid and calcium carbonate or sevelamer associated with a phosphorus-restricted diet. The sevelamer showed better results with a significant reduction in mortality from all causes. Final considerations: There was no consensus among the authors as to the gold standard therapy for patients with BMD-CKD in early stages.
\end{abstract}

Keywords: Mineral and bone disorder in chronic kidney disease, Chronic kidney failure, Secondary hyperparathyroidism.

\footnotetext{
1 Universidade Federal de São João Del Rei, Divinópolis - MG. *Email: kanq@bol.com.br

2 Hospital São João de Deus, Divinópolis - MG.
}

SUBMETIDO EM: 5/2020

ACEITO EM: 7/2020

PUBLICADO EM: 10/2020

REAS / EJCH | Vol.12(10) | e4067 | DOI: https://doi.org/10.25248/reas.e4067.2020 Página 1 de 13 


\section{RESUMEN}

Objetivo: Identificar la propuesta estándar de oro para la prevención y el tratamiento de los trastornos minerales y óseos en adultos y ancianos con enfermedad renal crónica no relacionada con la diálisis. Métodos: Una revisión sistemática de ensayos controlados aleatorios. Resultados: Se incluyeron ocho artículos: calcitriol o colecalciferol, con calcitriol que tiene un mejor resultado en la corrección de la hipocalcemia, reduciendo las fosfatasas alcalinas séricas y la hormona paratiroidea; alfacalcidol con control de la enfermedad ósea en el $42 \%$ de los pacientes; el colecalciferol aumentó el calcidiol sérico y disminuyó los niveles séricos de parathormona; colecalciferol durante un período prolongado con mejoría en la deficiencia de vitamina $D$ y disminución de los niveles séricos de parathormona; el alendronato durante mucho tiempo no alteró la progresión de la calcificación vascular; el acetato de calcio, el carbonato de lantano o el carbonato de sevelámer disminuyeron el fósforo urinario promedio y atenuaron la progresión del hiperparatiroidismo; El calcitriol asociado con la intervención de calcio en la dieta, el calcitriol aislado disminuyó los niveles de PTHi en ayunas y, cuando se combinó con calcio en la dieta, normalizó las respuestas posprandiales de calcemia y paratiroides y carbonato de calcio o sevelamer asociado con una dieta restringida en fósforo. El sevelamer mostró mejores resultados con una reducción significativa en la mortalidad por todas las causas. Consideraciones finales: No hubo consenso entre los autores en cuanto a la terapia estándar de oro para pacientes con DMO-ERC en etapas tempranas.

Palabras clave: Trastorno mineral y óseo asociado a la enfermedad renal crónica, Insuficiencia renal crónica, Hiperparatiroidismo secundario.

\section{INTRODUÇÃO}

A Doença Renal Crônica (DRC) é um importante problema de saúde pública, com taxa de prevalência crescente, afetando $10 \%$ da população adulta em todo o mundo e mais de um terço dos idosos (STENGEL B, et al., 2011). E em 2019, aproximadamente 139.631 pacientes se encontravam com diagnóstico de Doença Renal Crônica Terminal (DRCT) em tratamento com Terapia Renal Substitutiva (TRS)no Brasil. Esses pacientes têm suas capacidades laborais diminuídas, sofrem com a diminuição da qualidade de vida e ainda, oneram os cofres públicos com tratamento de alto custo (SBN, 2019).

Por definição a DRC consiste em anormalidades da estrutura e/ou função dos rins por três ou mais meses, com implicações para a saúde do paciente, podendo ser evidenciada em exames de imagem, sanguíneos e/ou urinários (KDIGO, 2012).

Nas fases iniciais a taxa de filtração glomerular estimada (TFGe) encontra-se preservada e as manisfestações clínicas são discretas ou mesmo ausentes e, em geral, a progressão para estágios mais avançados é insidiosa.

Com a evolução do quadro, os sinais e sintomas tornam-se mais evidentes e, caso não seja devidamente assistido, o paciente evolui naturalmente para a perda total da função renal (FR), quando passa a necessitar de TRS (BASTOS MG e KIRSZTAJN GM, 2011).

Paralelamente e associadas à progressão da perda da FR outras complicações podem surgir como, por exemplo, anemia, acidose metabólica, desnutrição e alteração do metabolismo mineral e ósseo. Essas complicações associadas à DRC, não controladas, geram comorbidades de difícil controle clínico (VALENTE LM, et al., 2015). No que diz respeito especificamente às complicações atribuídas ao desajuste das funções endócrinas e exócrinas dos rins destaca-se as alterações no metabolismo mineral e ósseo (KANBAY M, et al., 2013).

Essa alteração, recebe o nome de distúrbio mineral e ósseo da doença renal crônica (DMO-DRC) e manifesta-se por: alterações laboratoriais nos níveis séricos de cálcio, fósforo, paratormônio (PTH), ou metabolismo de vitamina D; alterações no remodelamento ósseo (mineralização, volume, crescimento linear ou força) e calcificação vascular ou de outros tecidos moles diagnosticados por exames de imagem (KDIGO, 2017; FILHO AJI e MELAMED ML, 2013; SPASOVISKI G, et al., 2012). 
Embora muito se tenha publicado sobre DMO-DRC em pacientes em TRS's, ainda são escassas as publicações sobre o manejo preventivo e clínico terapêutico desta condição nas fases precoces da DRC (ABDU A, et al., 2019; ZUMNAN MG, et al., 2018; JIN JJ, et al., 2018).

Diante do impacto na saúde geral da pessoa na iminência do DMO-DRC, acredita-se que quanto mais cedo houver intervenção, melhor poderá ser o prognóstico da evolução dessa condição dupla de saúde. Neste sentido, o objetivo deste estudo foi identificar proposta padrão ouro de prevenção e tratamento para o distúrbio mineral e ósseo em adultos e idosos com doença renal crônica (DRC) não dialítica.

\section{MÉTODOS}

Realizou-se uma revisão sistemática, com registro no PROSPERO 42017057933, baseada no guideline The PRISMA Statement (MOHER D, et al., 2009). Estabeleceu-se a estratégia "PICOS": "P"(patients): adultos e idosos com DRC nos estágios 3a, 3b, 4 e 5 não dialítico (ND) e com DMO; "I" (intervention): tratamento farmacológico ou não farmacológico para controle do DMO; "C" (control): adultos com DRC nos estágios 3a, 3b, 4 e 5 ND e com DMO que não participem da principal intervenção a ser testada; "O" (outcomes): controle do DMO com no mínimo os níveis séricos de cálcio e fósforo; "S" (study design) ensaios clínicos randomizados.

Foram considerados elegíveis estudos experimentais, sem restrição de idioma e data de publicação. Foram excluídos estudos experimentais com animais, sem grupo controle ou que não apresentaram os resultados das intervenções, artigos de revisão, observacionais, editoriais, cartas ao editor, notícias, comentários, relatórios de simpósios, dissertações, teses, resumos publicados em anais, que envolveram pacientes em TRS, nefrectomizados, transplantados e crianças.

As bases de dados utilizadas foram PubMed, Scopus e Biblioteca Virtual de Saúde (BVS) com estudos publicados até 20/01/2019. E o formato da busca conforme a base ocorreu da seguinte maneira: O Medical Subject Heading (MeSH) do PubMed foi utilizado para definir os descritores: "Chronic Kidney Disease", "Chronic Kidney Disease - Mineral and Bone Disorder", "Renal Insufficiency, Chronic".

A palavra "mineral metabolism", embora não esteja inserida no banco de descritores em saúde, foi introduzida na busca por ter representatividade no tema. Selecionou a opção "advanced search" e, em cada base de dados, foram realizadas quatro buscas: "Chronic Kidney Disease" AND "Chronic Kidney DiseaseMineral and Bone Disorder"; "Chronic Kidney Disease" AND "mineral metabolism"; "Renal Insufficiency, Chronic" AND "Chronic Kidney Disease-Mineral and Bone Disorder" e "Renal Insufficiency, Chronic" AND "mineral metabolism".

Após a seleção de todos os artigos realizou-se o cruzamento para retirada dos duplicados e as Variáveis coletadas foram: ano de publicação, país de realização do estudo, tamanho da amostra, média de idade e sexo dos participantes, estágios da DRC, parâmetros de determinação de DMO, intervenção para DMO, resultados.

Para agregar robustez a essa revisão foram realizadas análises adicionais: avaliação do risco de viés na qual foi utilizado a Cochrane Collaboration's tool, ${ }^{17,18}$ avaliando sete domínios: descrição de alocação randomizada, descrição de ocultação de sequência de alocação, cegamento de participantes e pesquisadores, cegamento da avaliação do desfecho, avaliação de dados incompletos para a determinação do desfecho, risco de seleção de desfechos e identificação de outros vieses.

Determinação de qualidade da evidência utilizando a metodologia GRADE classificando-as como alta (escore +4$)$, moderada (escore +3 ), baixa (escore +2 ) e qualidade muito baixa (pontuação +1$)$. É importante mencionar que todos os estudos incluídos eram ensaios clínicos randomizados e, portanto, a avaliação começou com a pontuação máxima, +4 . Os estudos que apresentaram médio risco de viés perderam um ponto e os estudos que apresentaram alto risco de viés perderam dois pontos (BALSHEMA H, et al., 2011). 


\section{RESULTADOS}

Foram identificados após a leitura do título e resumo, pelo pesquisador (KQ) 10 artigos e pelo outro pesquisador (JAA) 16 artigos. As divergências foram analisadas e, quando não houve consenso, (3 artigos), dois pesquisadores independentes (AO e AOB) os analisaram. Posteriormente, foram selecionados 14 artigos para leitura integral. A seguir, apenas oito estudos preencheram os critérios para serem incluídos na análise (Figura 1).

Figura 1 - Fluxograma das fases da revisão sistemática.

Artigos identificados através das quatro buscas em cada base de dados (1658)

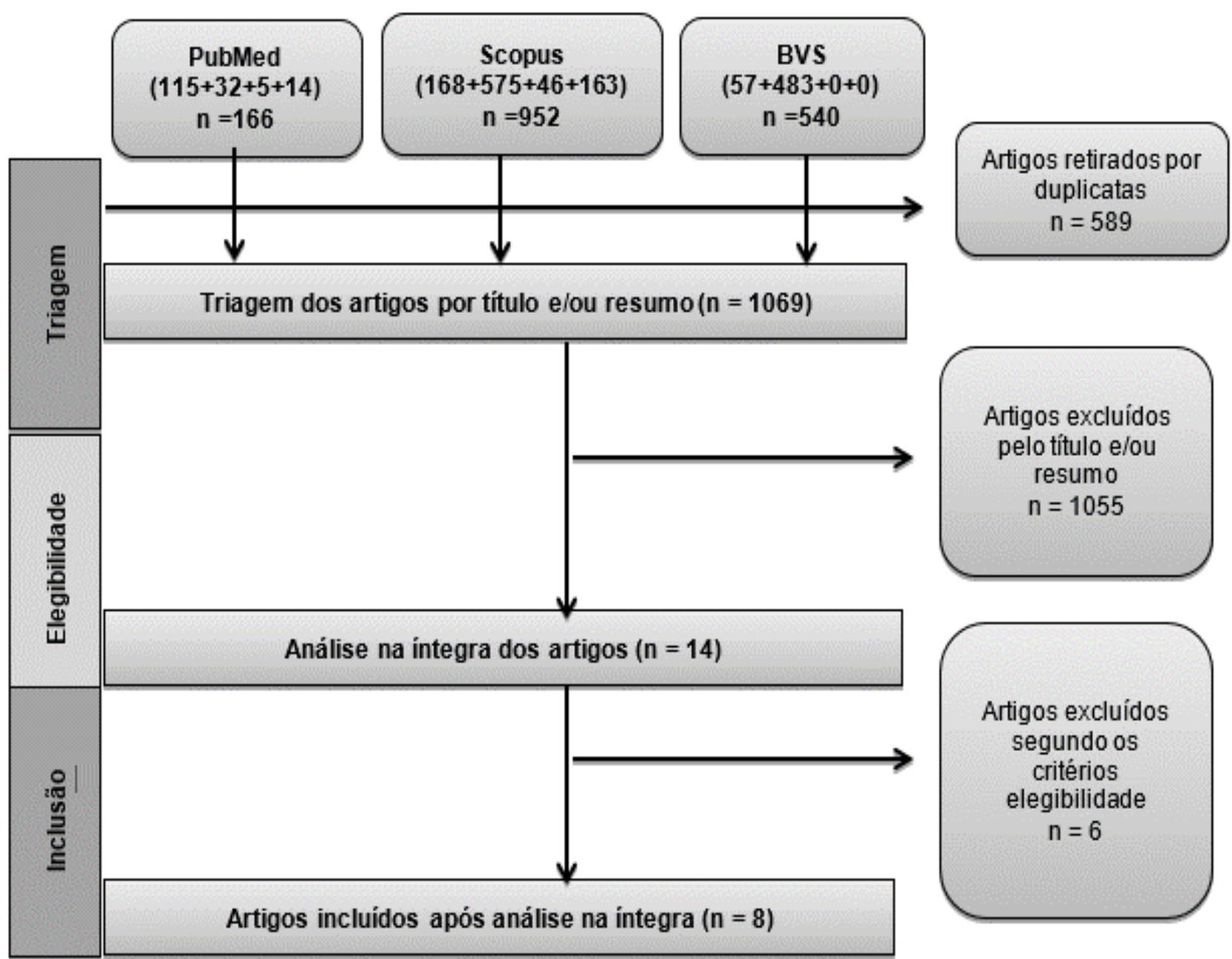

Fonte: Quadros KAN, et al., 2020. Fluxograma baseado no guideline PRISMA (Moher D, et al., 2009).

Os artigos foram publicados entre 1978 a 2015 nos continentes Europeu, Asiático e Americano. As variáveis coletadas nos estudos estão descritas no Quadro 1, sendo os resultados detalhados textualmente a seguir. 
Revista Eletrônica Acervo Saúde / Electronic Journal Collection Health ISSN 2178-2091

Quadro 1 - Estudos que identificaram intervenções para DMO em pacientes com DRC $(n=8)$.

\begin{tabular}{|c|c|c|c|c|c|c|c|c|c|}
\hline \multicolumn{2}{|c|}{ ESTUDO } & \multicolumn{6}{|c|}{ MÉTODO } & \multicolumn{2}{|r|}{ RESULTADO } \\
\hline & Autor/Ano/País & $\begin{array}{l}\text { Tamanho } \\
\text { amostral }\end{array}$ & $\begin{array}{l}\text { Parâmetros para } \\
\text { determinação da presença ou } \\
\text { ausência do Distúrbio Mineral } \\
\text { e Ósseo e /ou objetivo }\end{array}$ & $\begin{array}{l}\text { Média } \\
\text { de Idade } \\
\text { (anos) }\end{array}$ & $\%$ Sexo & $\begin{array}{l}\text { Classificação } \\
\text { do estágio da } \\
\text { DRC }\end{array}$ & $\begin{array}{l}\text { Intervenção/prevenção } \\
\text { para Distúrbio Mineral e } \\
\text { Ósseo }\end{array}$ & $\begin{array}{l}\text { Quando o } \\
\text { Distúrbio } \\
\text { Mineral e } \\
\text { Ósseo foi } \\
\text { identificado }\end{array}$ & Resultado da Intervenção \\
\hline 1 & $\begin{array}{l}\text { Christiansen C, } \\
\text { et al., 1978. [1] }\end{array}$ & $\begin{array}{l}\text { Total: } 18 \\
\text { Intervenção: } 9 \\
\text { Controle: } 9\end{array}$ & $\begin{array}{l}\text { Classificação de osteodistrofia } \\
\text { renal leve. Os pacientes que } \\
\text { preencheram pelo menos dois } \\
\text { dos três critérios seguintes } \\
\text { foram selecionados: conteúdo } \\
\text { mineral ósseo inferior a } 90 \% \text { do } \\
\text { normal, cálcio sérico inferior ao } \\
\text { normal, e fosfatases alcalinas } \\
\text { séricas maiores do que o } \\
\text { normal. }\end{array}$ & $\begin{array}{l}\text { Mulheres } \\
: 54 \text { anos } \\
\text { Homens: } \\
44 \text { anos }\end{array}$ & $\begin{array}{l}27 \% \\
\text { Homens } \\
72 \% \\
\text { Mulheres }\end{array}$ & $\begin{array}{l}\text { Pacientes } \text { não } \\
\text { dialisados } \\
\text { depuraçãa da } \\
\text { creatinina abaixo } \\
\text { de } 35 \mathrm{ml} / \mathrm{min} \text {. }\end{array}$ & $\begin{array}{l}\text { Os } 18 \text { pacientes foram } \\
\text { alocados aleatoriamente } \\
\text { para dois subgrupos: um } \\
\text { foi tratado com calcitriol 1, } \\
25 \text { (OH) 2D3 (grupo } \\
\text { I) } 1 \text { micrograma, o outro/ } \\
\text { (grupo II) com vitamina D3 } \\
4000 \mathrm{UI} \text { (calcidiol). Todos } \\
\text { os pacientes receberam } \\
\text { diariamente 0,5 g de } \\
\text { cálcio. }\end{array}$ & $\begin{array}{l}\text { Após a } \\
\text { investigação } \\
\text { primária, os } \\
\text { pacientes } \\
\text { foram } \\
\text { observados } \\
\text { por seis } \\
\text { meses para } \\
\text { determinar o } \\
\text { curso da } \\
\text { doença }\end{array}$ & $\begin{array}{l}\text {-O uso da } 1,25(\mathrm{OH}) 2 \mathrm{D} 3 \text { corrigiu a hipocalcemia, } \\
\text { reduziu as fosfatases alcalinas séricas e } \\
\text { hormônio paratireóideo } \\
\text { - O uso da D3 teve efeitos semelhantes aqueles } \\
\text { referidos acima, mas menos pronunciados. } \\
\text { A deterioração da função renal foi uma grande } \\
\text { limitação do uso clínico de } 1,25(\mathrm{OH}) 2 \mathrm{D} 3 \text { e D3 } \\
\text { em pacientes não dialisados. }\end{array}$ \\
\hline 2 & $\begin{array}{l}\text { Hamdy NA, et } \\
\text { al., } \quad 1995 . \\
\text { Bélgica, França, } \\
\text { Holanda e Reino } \\
\text { Unido [2] }\end{array}$ & $\begin{array}{l}\text { Total } 176 \\
\text { Intervenção: } \\
89 \\
\text { Controle: } 87\end{array}$ & $\begin{array}{l}\text { Abordagem preventiva na fase } \\
\text { inicial do DMO-DRC: Ausência } \\
\text { do DMO-DRC: Sem evidência } \\
\text { clínica, bioquímica ou } \\
\text { radiográfica de doença óssea }\end{array}$ & $\begin{array}{l}\text { Média de } \\
\text { idade: } 53 \\
\text { anos no } \\
\text { grupo } \\
\text { intervenç } \\
\text { ão e } 51 \\
\text { anos no } \\
\text { grupo } \\
\text { controle. }\end{array}$ & $\begin{array}{l}54 \% \\
\text { homens } \\
\text { no grupo } \\
\text { intervençã } \\
\text { o e } 53 \% \\
\text { homens } \\
\text { no grupo } \\
\text { controle. }\end{array}$ & $\begin{array}{l}\text { DRC leve a } \\
\text { moderada: } \\
\text { Depuração da } \\
\text { creatinina } 15-50 \\
\mathrm{ml} / \mathrm{min} \text { ) }\end{array}$ & \begin{tabular}{llr} 
Alfacalcidol & \multicolumn{2}{r}{0,25} \\
microgramas & (titulado de \\
acordo & com & a \\
concentração & sérica de \\
cálcio) ou placebo \\
administrado por dois \\
anos.
\end{tabular} & $\begin{array}{l}\text { Trata-se da } \\
\text { abordagem } \\
\text { para } \\
\text { Prevenção } \\
\text { primária do } \\
\text { DMO. }\end{array}$ & $\begin{array}{l}\text { No grupo controle a fosfatase alcalina no soro e } \\
\text { a concentração de hormônio da paratireóide } \\
\text { aumentaram } 13 \% \text { e } 126 \% \text {, respectivamente, nos } \\
\text { controles, mas não alteraram em pacientes com } \\
\text { alfacalcidol ( } P<0,001) \text {. } \\
\text { - Os índices histológicos do turnover ósseo } \\
\text { melhoraram significativamente nos pacientes } \\
\text { com alfacalcidol. A doença óssea foi resolvida } \\
\text { em } 42 \% \text { daqueles que receberam alfacalcidol em } \\
\text { comparação com placebo. }\end{array}$ \\
\hline 3 & $\begin{array}{l}\text { Dogan E, et al., } \\
2008 . \text { Turquia. } \\
{[3]}\end{array}$ & $\begin{array}{l}\text { Total } 40 \\
\text { Intervenção: } \\
20 \\
\text { Controle: } 20\end{array}$ & $\begin{array}{l}\text { Avaliar os efeitos da } \\
\text { suplementação do colecalciferol } \\
\text { nos níveis de PTHi e fosfatase } \\
\text { alcalina. Foram excluídos: } \\
\text { pacientes com PTHi<200; Ca> } \\
10,5 \mathrm{mg} / \mathrm{dL} ; \mathrm{P}>6,5 \mathrm{mg} / \mathrm{dL} \text {, ou } \\
\text { aqueles que ingeriram qualquer } \\
\text { droga de vitamina } \mathrm{D} \text { ou podem } \\
\text { ser afetados pelo metabolismo } \\
\text { das vitaminas no organismo, } \\
\text { durante pelo menos um mês }\end{array}$ & $\begin{array}{l}\text { Idade } \\
\text { média de } \\
49+/-14\end{array}$ & $\begin{array}{l}50 \% \\
\text { Homens }\end{array}$ & $\begin{array}{l}\text { TFG entre } 29 \text { e } \\
60 \mathrm{~mL} / \mathrm{min}\end{array}$ & $\begin{array}{l}\text { Dose única de } 300.000 \text { UI } \\
\text { de vitamina D } \\
\text { colecalciferol/por mês. } \\
\text { Grupo controle não tomou } \\
\text { nenhum remédio com } \\
\text { vitamina D ou foram } \\
\text { afetados pelo metabolismo } \\
\text { da vitamina D no } \\
\text { organismo durante um } \\
\text { mês }\end{array}$ & $\begin{array}{l}\text { Prevenção } \\
\text { primária do } \\
\text { DMO. }\end{array}$ & $\begin{array}{l}\text {-O calcidiol sérico dos pacientes do grupo de } \\
\text { tratamento aumentou significativamente }(6,8+/- \\
3,5 \text { a } 17,8+/-21,4 \mathrm{ng} / \mathrm{mL}, \mathrm{p}<0,001) \text { e o nível de } \\
\text { PTHi diminuiu significativamente no grupo de } \\
\text { tratamento (368 +/- } 274 \text { a } 279+/-179 \mathrm{pg} / \mathrm{ml}, \mathrm{p} \\
<0,001) \text {. } \\
\text { - O tratamento de colecalciferol oral causou uma } \\
\text { diminuição significante do nível de PTH no soro, } \\
\text { mas não causou uma alteração estatisticamente } \\
\text { significante no Ca, } P \text {, proporção de CaxP ou taxa } \\
\text { de creatinina urinária de cálcio na DRC. }\end{array}$ \\
\hline
\end{tabular}

REAS / EJCH | Vol.12(10) | e4067 | DOI: https://doi.org/10.25248/reas.e4067.2020 Página 5 de 13 


\section{Revista Eletrônica Acervo Saúde / Electronic Journal Collection Health ISSN 2178-2091}

\begin{tabular}{|c|c|c|c|c|c|c|c|c|c|}
\hline \multicolumn{2}{|c|}{ ESTUDO } & \multicolumn{6}{|c|}{ MÉTODO } & \multicolumn{2}{|r|}{ RESULTADO } \\
\hline & Autor/Ano/País & $\begin{array}{l}\text { Tamanho } \\
\text { amostral }\end{array}$ & $\begin{array}{l}\text { Parâmetros para } \\
\text { determinação da presença ou } \\
\text { ausência do Distúrbio Mineral } \\
\text { e Ósseo e /ou objetivo }\end{array}$ & $\begin{array}{l}\text { Média } \\
\text { de Idade } \\
\text { (anos) }\end{array}$ & $\%$ Sexo & $\begin{array}{l}\text { Classificação } \\
\text { do estágio da } \\
\text { DRC }\end{array}$ & $\begin{array}{l}\text { Intervenção/prevenção } \\
\text { para Distúrbio Mineral e } \\
\text { Ósseo }\end{array}$ & $\begin{array}{c}\text { Quando o } \\
\text { Distúrbio } \\
\text { Mineral e } \\
\text { Ósseo foi } \\
\text { identificado }\end{array}$ & Resultado da Intervenção \\
\hline 4 & $\begin{array}{l}\text { Oksa A, et al., } \\
2008 . \\
\text { Eslovaquia [4] }\end{array}$ & $\begin{array}{l}\text { Total } 87 \\
\text { Grupo A } 44 \\
\text { Grupo B } 43\end{array}$ & $\begin{array}{l}\text { Avaliou os efeitos do tratamento } \\
\text { de } 12 \text { meses com vitamina } \mathrm{D}(3) \\
\text { sobre o metabolismo mineral e } \\
\text { hormônios calciotrópicos em } \\
\text { pacientes com DRC estágios 2- } \\
4 \text {. } \\
\text { No início do estudo foi aferido } \\
\text { PTHi, Cálcio, fósforo, } 25 \text { (OH) D } \\
3 \\
\text { (25-Hydroxy Vitamin D), e } 1,25 \\
\text { (OH) 2D3 (1,25- } \\
\text { Dihydroxy Vitamina D. Foram } \\
\text { excluídos pacientes com } \\
\text { tratamentos concomitantes que } \\
\text { interferem no metabolismo } \\
\text { mineral forma excluídos, exceto } \\
\text { aqueles em uso de } \\
\text { carbonato de cálcio para a } \\
\text { correção da acidose metabólica. }\end{array}$ & $\begin{array}{l}\text { Idade } \\
\text { média } 66 \\
\text { anos }\end{array}$ & $\begin{array}{l}38 \% \\
\text { Mulheres }\end{array}$ & $\begin{array}{l}\text { TFG estável de } \\
15 \text { a } 89 \mathrm{ml} / \mathrm{min} / \\
1,73 \mathrm{~m}^{2} / \\
\text { Estágios } 2 \text { a } 4\end{array}$ & $\begin{array}{l}\text { Tratamento com } \\
\text { colecalciferol oral com } \\
5.000 \text { UI / semana (grupo } \\
\text { A) ou } 20.000 \text { UI / semana } \\
\text { (grupo B), por um ano. }\end{array}$ & $\begin{array}{l}\text { Prevenção } \\
\text { primária do } \\
\text { DMO. Foi } \\
\text { identificado } \\
\text { níveis } \\
\text { séricos de } \\
\text { PTHi, } \\
\text { Cálcio, } \\
\text { Fosforo e } \\
\text { vitamina D } \\
\text { no início do } \\
\text { estudo (linha } \\
\text { de base) }\end{array}$ & $\begin{array}{l}\text { - A deficiência de vitamina } \mathrm{D} \text { foi revelada em } 72 \\
(83 \%) \text { pacientes na linha de base e } 37(43 \%) \text { no } \\
\text { mês } 12 \text {. Os níveis de } 25(\mathrm{OH}) \mathrm{D}(3) \text { aumentaram } \\
\text { mais com a dose mais alta. } \\
\text { - As concentrações de hormônio paratireoidiano } \\
\text { (PTH) foram reduzidas. } \\
\text { - A deficiência de vitamina D na DRC melhorou } \\
\text { significativamente após o tratamento com } \\
\text { colecalciferol a } 12 \text { meses, sendo a dose alta mais } \\
\text { eficaz e igualmente segura. }\end{array}$ \\
\hline 5 & $\begin{array}{l}\text { ToussaintND, et } \\
\text { al., } \quad 2010 . \\
\text { Austrália [6] }\end{array}$ & $\begin{array}{l}\text { Total: } 50 \\
\text { Intervenção: } \\
25 \\
\text { Controle: } 25\end{array}$ & $\begin{array}{l}\text { Pacientes com diagnóstico } \\
\text { prévio de Calcificação Vascular }\end{array}$ & $\begin{array}{l}\text { Idade } \\
\text { média } \\
\text { basal foi } \\
\text { de } 63.1 \\
+/-1.8 \\
\text { anos }\end{array}$ & $\begin{array}{l}65 \% \\
\text { homens }\end{array}$ & $\begin{array}{ll}\text { Estágios } & 3 \text { e } 4 \\
\text { (TFG: } & 20-60 \\
\mathrm{~mL} / \mathrm{min} / & \\
1.73 \mathrm{~m}^{2} \text { ) } & \end{array}$ & $\begin{array}{l}\text { Grupo Intervenção: } \\
\text { recebeu Alendronato } 70 \\
\mathrm{mg} / \mathrm{kg} \text {, via oral, } \\
\text { semanalmente por } 18 \\
\text { meses, enquanto o grupo } \\
\text { controle recebeu placebo. }\end{array}$ & Não relatado & $\begin{array}{l}\text { Em 18 meses, não houve diferença na } \\
\text { progressão da calcificação vascular com } \\
\text { alendronato em comparação com o placebo. } \\
\text { - Houve aumento da densidade mineral da } \\
\text { coluna lombar e uma tendência para uma melhor } \\
\text { velocidade da onda de pulso com alendronato. } \\
\text { - A densidade mineral femoral foi semelhante } \\
\text { entre os grupos. - O alendronato não diminuiu a } \\
\text { progressão da calcificação vascular em em } \\
\text { comparação com placebo em pacientes com } \\
\text { DRC durante } 18 \text { meses. }\end{array}$ \\
\hline 6 & $\begin{array}{l}\text { Block GA, et al., } \\
\text { 2012. EUA [5] }\end{array}$ & $\begin{array}{l}\text { Total } 118 \\
\text { Grupo A } 30 \\
\text { Grupo B } 30 \\
\text { Grupo C } \\
30 \\
\text { Placebo } 58\end{array}$ & $\begin{array}{l}\text { Determinar a eficácia dos } \\
\text { aglutinantes de fosfato em } \\
\text { pacientes com DRC moderada e } \\
\text { concentração normal ou quase } \\
\text { normal de Fósforo sérico } \geq 3,5 \text { e } \\
<6,0 \mathrm{mg} / \text { dl }\end{array}$ & $\begin{array}{l}\text { Idade } \\
\text { média } \\
\text { dos } \\
\text { grupos } \\
\text { intervenç } \\
\text { ões } \\
68 \pm 11\end{array}$ & $\begin{array}{l}50 \% \\
\text { Homens } \\
\text { nos } \\
\text { grupos } \\
\text { intervençõ } \\
\text { es }\end{array}$ & $\begin{array}{l}\text { DRC moderada } \\
\text { a avançada - } \\
\text { TFG entre } 20 \mathrm{e} \\
45 \mathrm{ml} / \mathrm{min} \text { por } \\
1,73 \mathrm{~m}^{2}\end{array}$ & $\begin{array}{l}\text { Tratamento com acetato } \\
\text { de cálcio = } 667 \mathrm{mg} \text {, } \\
\text { carbonato de lantânio = } \\
500 \mathrm{mg} \text { e carbonato de } \\
\text { sevelamer }=800 \mathrm{mg} \text { ou } \\
\text { placebo. }\end{array}$ & $\begin{array}{l}\text { Prevenção } \\
\text { primária do } \\
\text { DMO. }\end{array}$ & $\begin{array}{l}\text { - O fósforo sérico diminuiu de uma média basa } \\
\text { de } 4,2 \mathrm{mg} / \mathrm{dl} \text { nos braços ativo e placebo para } 3,9 \\
\mathrm{mg} / \mathrm{dl} \text { com terapia ativa e } 4,1 \mathrm{mg} / \mathrm{dl} \mathrm{com} \\
\text { placebo ( } \mathrm{P}=0,03 \text { ). } \\
\text { - Os aglutinantes de fosfato, mas não o placebo, } \\
\text { diminuíram em } 22 \% \text { o fósforo médio de urina de } \\
24 \text { horas. Os aglutinantes de fosfato diminuíram } \\
\text { significativamente no soro e o fósforo urinário } \\
\text { atenuaram a progressão do hiperparatireoidismo }\end{array}$ \\
\hline
\end{tabular}

REAS / EJCH | Vol.12(10) | e4067 | DOI: https://doi.org/10.25248/reas.e4067.2020 Página 6 de 13 


\section{Revista Eletrônica Acervo Saúde / Electronic Journal Collection Health ｜ISSN 2178-2091}

\begin{tabular}{|c|c|c|c|c|c|c|c|c|c|}
\hline \multicolumn{2}{|c|}{ ESTUDO } & \multicolumn{6}{|c|}{ MÉTODO } & \multicolumn{2}{|r|}{ RESULTADO } \\
\hline & Autor/Ano/País & $\begin{array}{l}\text { Tamanho } \\
\text { amostral }\end{array}$ & $\begin{array}{l}\text { Parâmetros para } \\
\text { determinação da presença ou } \\
\text { ausência do Distúrbio Mineral } \\
\text { e Ósseo e /ou objetivo }\end{array}$ & $\begin{array}{l}\text { Média } \\
\text { de Idade } \\
\text { (anos) }\end{array}$ & $\%$ Sexo & $\begin{array}{l}\text { Classificação } \\
\text { do estágio da } \\
\text { DRC }\end{array}$ & $\begin{array}{l}\text { Intervenção/prevenção } \\
\text { para Distúrbio Mineral e } \\
\text { Ósseo }\end{array}$ & $\begin{array}{l}\text { Quando o } \\
\text { Distúrbio } \\
\text { Mineral e } \\
\text { Ósseo foi } \\
\text { identificado }\end{array}$ & Resultado da Intervenção \\
\hline 7 & $\begin{array}{l}\text { Isakova T, et al., } \\
\text { 2012. EUA. [7] }\end{array}$ & $\begin{array}{l}\text { Total: } 24 \\
\text { Intervenção: } \\
12 \\
\text { Controle: } 12\end{array}$ & $\begin{array}{l}\text { Avaliou as flutuações diárias dos } \\
\text { metabólitos minerais } \\
\text { Em pacientes com DRC antes e } \\
\text { após o tratamento com calcitriol } \\
\text { de curta duração e testaram os } \\
\text { efeitos dos } \\
\text { suplementação de cálcio e } \\
\text { calcitriol sobre esses parâmetros } \\
\text { no cenário pós-prandial } \\
\text { dinâmico. } \\
\text { Inclusão: DRC com } \\
\text { Normocalcemia, } \\
\text { normofosfatemia e } \\
\text { hidroxivitamina D (25D) } \geq 30 \mathrm{ng} \text { / } \\
\text { ml. }\end{array}$ & $\begin{array}{l}\text { Intervenç } \\
\text { ão: } 60 \pm 7 \\
\text { Controle: } \\
55 \pm 9\end{array}$ & $\begin{array}{l}75 \% \\
\text { Homens } \\
25 \% \\
\text { Mulheres }\end{array}$ & Estágios 3 e 4 & $\begin{array}{l}\text { Calcitriol }(0,25 \mu \mathrm{g} \text { por dia } \\
\text { por uma semana) e } \\
\text { intervenção dietética de } \\
\text { cálcio ( } 250 \text { versus } 500 \mathrm{mg}) \\
\text { no café da manhã. }\end{array}$ & $\begin{array}{l}\text { Prevenção } \\
\text { primária do } \\
\text { DMO. }\end{array}$ & $\begin{array}{l}\text { O calcitriol diminuiu os níveis PTHi em jejum e, } \\
\text { quando combinado com carga de cálcio na dieta, } \\
\text { normalizou as respostas pós-prandiais de } \\
\text { calcemia e paratireóide. }\end{array}$ \\
\hline 8 & $\begin{array}{l}\text { Russo D, et al., } \\
\text { 2015. Itália [8] }\end{array}$ & $\begin{array}{l}\text { Total: } 113 \\
\text { Grupo } \\
\text { Carbonato de } \\
\text { Cálcio: } 47 \\
\text { Grupo } \\
\text { sevelamer: } 66\end{array}$ & $\begin{array}{l}\text { Diagnóstico da Calcificação } \\
\text { arterial coronariana na linha de } \\
\text { base }\end{array}$ & $\begin{array}{l}\text { Grupo } \\
\text { Carbonat } \\
\text { o de } \\
\text { Cálcio: } \\
58.4 \quad \pm \\
12.5 \\
\text { Grupo } \\
\text { sevelam } \\
\text { er: } \\
56.9 \quad \pm \\
11.5\end{array}$ & $\begin{array}{l}\text { Grupo } \\
\text { Carbonato } \\
\text { de Cálcio } \\
\text { (H/M)28/1 } \\
\text { Grupo } \\
\text { sevelamer } \\
: \\
(\mathrm{H} / \mathrm{M}) \\
45 / 21\end{array}$ & Estágio 3-4 & $\begin{array}{l}\text { Carbonato de cálcio ou } \\
\text { sevelamer adicionados à } \\
\text { dieta restrita ao fósforo }\end{array}$ & Não relatado & $\begin{array}{l}\text { Tratamento com carbonato de cálcio foi } \\
\text { associado ao aumento da progressão da } \\
\text { calcificação arterial coronariana (CAC) e à } \\
\text { ocorrência de mortalidade por todas as causas, } \\
\text { iniciação de diálise e ponto final composto } \\
\text { - Redução significativa na mortalidade por todas } \\
\text { as causas, iniciação de diálise e risco de ponto } \\
\text { final composto foi alcançada, combinando dieta } \\
\text { restrita de fósforo e sevelamer em pacientes com } \\
\text { DRC não dialisados com progressão de } \\
\text { CAC ausente ou moderada, mas não acelerada. }\end{array}$ \\
\hline
\end{tabular}

Fonte: Quadros KAN, et al., 2020.

REAS / EJCH | Vol.12(10) | e4067 | DOI: https://doi.org/10.25248/reas.e4067.2020 Página 7 de 13 
Em relação aos resultados dos tratamentos, Christiansen C, et al. (1980) obtiveram melhora de hipocalcemia, e redução dos níveis séricos da fosfatase alcalina sérica e do PTH com o uso do calcitriol. Já com uso do colecalciferol, a vitamina $\mathrm{D}$ inativa, obteve-se resultados semelhantes, mas menos pronunciados. Ainda nesse estudo, os resultados mostraram que após o uso do colecalciferol e do calcitriol os pacientes tiveram redução acelerada da perda da função renal (CHRISTIANSEN C, et al., 1980). Em três dos estudos o uso do colecalciferol, calcitriol e aglutinantes de fosfato promoveu a diminuição significante do nível de PTH (DOGAN E, et al., 2008; BLOCK GA, et al., 2012; ISAKOVA T, et al., 2012).

Em um estudo que utilizou o alfacalcidol, (análogo da vitamina D3 inativa) a doença óssea foi resolvida em $42 \%$ em comparação com placebo (HAMDY NA, et al., 1995). No estudo de Oksa A, et al. (2008) a deficiência de vitamina D na DRC melhorou significativamente após o tratamento com colecalciferol por 12 meses, sendo a dose alta mais eficaz e igualmente segura (OKSA A, et al., 2008). Os aglutinantes de fosfato (quelantes do fósforo) diminuíram significativamente os níveis de fósforo, porém, eles também promoveram a progressão da doença cardiovascular (DCV) (BLOCK GA, et al., 2012). Já o alendronato utilizado para tratar a osteoporose, em especial em mulheres pós menopausa, no estudo de Toussainte ND, et al. (2010) foi utilizado para avaliar efeito dos bifosfonatos na DCV em pacientes com DRC e demonstrou que não houve diminuição na progressão da DCV em pacientes com DMO associado.

Do total de oito estudos, três apresentaram alto risco de viés, três médio risco de viés (indeterminado) e dois baixo risco de viés conforme descrito na Tabela 1.

Tabela 1 - Avaliação do risco de viés utilizando a Cochrane Collaboration's tool.

\begin{tabular}{|c|c|c|c|c|c|c|c|c|}
\hline Estudos & $\begin{array}{l}\text { Alocaçã } \\
\text { o } \\
\text { randomi } \\
\text { zada }\end{array}$ & $\begin{array}{l}\text { Ocultação } \\
\text { de } \\
\text { sequência } \\
\text { de } \\
\text { alocação }\end{array}$ & $\begin{array}{c}\text { Cegamento } \\
\text { de } \\
\text { pesquisadore } \\
\text { s e } \\
\text { participantes }\end{array}$ & $\begin{array}{l}\text { Cegament } \\
\text { o da } \\
\text { avaliação } \\
\text { de } \\
\text { desfecho }\end{array}$ & $\begin{array}{c}\text { Dados } \\
\text { incompletos } \\
\text { para o } \\
\text { desfecho }\end{array}$ & $\begin{array}{l}\text { Risco de } \\
\text { seleção de } \\
\text { desfechos }\end{array}$ & $\begin{array}{l}\text { Outros } \\
\text { vieses }\end{array}$ & $\begin{array}{c}\text { Risco de } \\
\text { viés } \\
\text { sumarizad } \\
0\end{array}$ \\
\hline $\begin{array}{l}\text { Christiansen } \\
\text { C, et al., } \\
1978\end{array}$ & + & $?$ & - & - & + & + & + & Alto \\
\hline $\begin{array}{l}\text { Hamdy NA, } \\
\text { et al., } 1995\end{array}$ & + & $?$ & + & + & + & + & + & Médio \\
\hline $\begin{array}{l}\text { Dogan E, et } \\
\text { al., } 2008\end{array}$ & + & $?$ & + & $?$ & + & + & + & Médio \\
\hline $\begin{array}{l}\text { Toussaint } \\
\text { ND, et al., } \\
2010\end{array}$ & + & + & + & + & + & + & + & Baixo \\
\hline $\begin{array}{l}\text { Okša A, et } \\
\text { al., } 2008\end{array}$ & + & $?$ & - & - & + & + & + & Alto \\
\hline $\begin{array}{l}\text { Block GA, et } \\
\text { al., } 2012\end{array}$ & + & + & + & + & + & + & + & Baixo \\
\hline $\begin{array}{l}\text { Russo D, et } \\
\text { al., } 2015\end{array}$ & + & $?$ & + & + & + & + & + & Médio \\
\hline $\begin{array}{l}\text { Isakova T, et } \\
\text { al., } 2012\end{array}$ & + & $?$ & - & - & + & + & + & Alto \\
\hline
\end{tabular}

Legenda: +: Baixo risco de viés; ?: Risco indeterminado de viés; - :Alto risco de viés.

Fonte: Quadros KAN, et al., 2020. 
Quanto à qualidade da evidência, dois estudados mostraram alta qualidade de evidência, quatro média qualidade de evidência e dois baixa qualidade de evidência (Tabela 2).

Tabela 2- Avaliação da qualidade de evidência utilizando a metodologia GRADE.

\begin{tabular}{|c|c|c|c|c|c|c|c|c|c|c|}
\hline Estudos & $\begin{array}{c}\text { Risco } \\
\text { de viés }\end{array}$ & $\begin{array}{c}\text { Inconsis } \\
\text { tência }\end{array}$ & $\begin{array}{c}\text { Relação } \\
\text { indireta } \\
\text { entre } \\
\text { exposição } \\
\text { e desfecho }\end{array}$ & $\begin{array}{c}\text { Impre } \\
\text { cisão }\end{array}$ & $\begin{array}{c}\text { Viés de } \\
\text { Publica } \\
\text { ção }\end{array}$ & $\begin{array}{c}\text { Gran } \\
\text { de } \\
\text { efeit } \\
\text { o }\end{array}$ & $\begin{array}{c}\text { Gradie } \\
\text { nte } \\
\text { dose- } \\
\text { respos } \\
\text { ta }\end{array}$ & $\begin{array}{c}\text { Análise de } \\
\text { confundid } \\
\text { ores } \\
\text { residuais }\end{array}$ & $\begin{array}{c}\text { Tot } \\
\text { al }\end{array}$ & $\begin{array}{c}\text { Quali } \\
\text { dade } \\
\text { da } \\
\text { Evidê } \\
\text { ncia }\end{array}$ \\
\hline $\begin{array}{c}\text { Christians } \\
\text { en C, et } \\
\text { al., 1978 }\end{array}$ & -2 & 0 & 0 & 0 & 0 & 0 & 0 & 0 & +2 & Baixa \\
\hline $\begin{array}{c}\text { Hamdy } \\
\text { NA, et al., } \\
\text { 1995 }\end{array}$ & -1 & 0 & 0 & 0 & 0 & 0 & 0 & 0 & +3 & Média \\
\hline $\begin{array}{c}\text { Dogan E, } \\
\text { et al., 2008 }\end{array}$ & -1 & 0 & 0 & 0 & 0 & 0 & 0 & 0 & +3 & Média \\
\hline $\begin{array}{c}\text { Toussaint } \\
\text { ND, et al., } \\
\text { 2010 }\end{array}$ & 0 & 0 & 0 & 0 & 0 & 0 & 0 & 0 & +4 & Alta \\
\hline $\begin{array}{c}\text { Okša A, et } \\
\text { al., 2008 }\end{array}$ & -2 & 0 & 0 & 0 & 0 & 0 & +1 & 0 & +3 & Média \\
\hline $\begin{array}{c}\text { Block GA, } \\
\text { et al., 2012 }\end{array}$ & 0 & 0 & 0 & 0 & 0 & 0 & 0 & 0 & +4 & Alta \\
\hline $\begin{array}{c}\text { Russo D, } \\
\text { t al., 2015 }\end{array}$ & -1 & 0 & 0 & 0 & 0 & 0 & 0 & 0 & +3 & Média \\
\hline $\begin{array}{c}\text { Isakova T, } \\
\text { et al., 2012 }\end{array}$ & -2 & 0 & 0 & 0 & 0 & 0 & 0 & 0 & +2 & Baixa \\
\hline
\end{tabular}

Fonte: Quadros KAN, et al., 2020.

\section{DISCUSSÃO}

Entre os tratamentos para DMO-DRC utilizados, os estudos selecionados que apresentaram melhores resultados foram referidos por Christiansen C, et al. (1980) onde doses de Calcitriol (1,25 (OH)2 D3), de vitamina D3 (colecalciferol) associadas a 0,5 gramas de cálcio revelaram melhora da hipocalcemia, diminuição dos níveis séricos da fosfatase alcalina e do PTH. Sendo que com o calcitriol foi mais potente do que com o uso de vitamina D3. Por outro lado, embora tenham apresentado melhora no quadro do DMO-DRC, os pacientes que usaram o calcitriol desenvolveram simultaneamente a hipercalcemia, não sendo possível avaliar associação dessa alteração com a ingesta simultânea de 0,5 gramas de cálcio proposta para ambos os grupos.

De qualquer forma, esse é um achado preocupante no sentido de que o DMO, inclui a hipercalcemia, como fator de risco para a calcificação vascular (CV) e consequentemente para a DCV, conhecida causa crucial de morte em pacientes com DRC (BLOCK GA, et al., 2004) O mecanismo da CV consiste em um processo ativo de precipitação de cálcio e fósforo e também na presença de um desequilíbrio entre fatores estimuladores e inibidores da calcificação.

Em um importante estudo com 40.000 pacientes renais crônicos foi demonstrado que a hiperfosfatemia $(>5 \mathrm{mg} / \mathrm{dL})$, a hipercalcemia $(>9,0 \mathrm{mg} / \mathrm{dL})$ e o hiperparatireoidismo secundário à $\mathrm{DRC}$, retratado pelo PTH $>600 \mathrm{pg} / \mathrm{mL}$, foram preditores independentes de mortalidade geral (ROETKER NS, et al., 2019). O impacto da calcificação na mortalidade cardiovascular nos pacientes renais, não está apenas na complicação das lesões ateroscleróticas, mas também no aumento da rigidez da parede das artérias, o que afeta a contratilidade e perfusão miocárdica (GUÉRIN AP, et al., 2000; ADRAGÃO T, et al., 2004).

Diante desse cenário, é inquietante vislumbrar que embora controlados os principais marcadores desencadeadores do DMO-DRC, o desequilíbrio nos níveis de cálcio, poderá potencializar o agravamento de uma complicação associada, ou seja, a DCV. Não bastasse o desafio do controle do DMO-DRC per si, outro achado alarmante foi que esses mesmos autores identificaram uma rapidez maior na perda da FR após início 
do tratamento, em especial no grupo que usou o calcitriol. Essa perda rápida da FR torna efetivo um ciclo vicioso de descontrole total, pois ao se tratar o DMO causado pela DRC, pode além de potencializar o risco de DCV, piorar a função renal conduzindo o paciente a uma rápida evolução para fase terminal da DRC.

Por fim, os resultados deixam claro o desafio dos ajustes de doses e dos medicamentos, já que a relação risco-benefício em cada uma dessas instâncias é extremamente tênue e pode, ao mesmo tempo, controlar uma condição de saúde, e concomitantemente, descompensar outra. E ainda é preciso refletir se os efeitos benéficos do uso do calcitriol para o controle do metabolismo levemente alterado nas fases iniciais da DRC, quando a FR ainda está preservada, justificam seu uso, em detrimento da aceleração da diminuição da taxa de filtração glomerular alterada (TFGe).

Numa tentativa de subsidiar a abordagem a pacientes com DMO-DRC o Ministério da Saúde (2017) e as diretrizes Clínicas para Diagnóstico, Evolução, Prevenção e Tratamento do DMO-DRC (Clinical Practice Guideline Update for the Diagnosis, Evaluation, Prevention, and Treatment of Chronic Kidney Disease Mineral and Bone Disorder (KDIGO, 2017) criaram protocolos para essa condução na expectativa de adesão dos profissionais de saúde. Em estudo desenvolvido por Roetker NS, et al. (2019) envolvendo um total 799.300 pacientes, os autores avaliaram a aderência KDIGO (2017) no cumprimento da aferição dos níveis de PTH, cálcio, fósforo e vitamina $D$.

Os resultados mostraram que o acompanhamento dos marcadores bioquímicos do DMO-DRC estava aquém do ideal. Ou seja, ao que parece, nos estágios iniciais da DRC, o controle do DMO não é foco de abordagem preventiva.

Retomando novamente o estudo de Christiansen C, et al. (1980) embora tenha sido publicado há alguns anos, chama a atenção para um tema de discussão que ainda é atual, que é a administração dos análogos da vitamina D como forma indireta de controle do PTH. Essa falta de consenso do padrão ouro para controle do DMO-DRC talvez justifique ainda hoje a atual recomendação de não se utilizar a vitamina $D$, para controle indireto do DMO e usá-la apenas em suspeita ou confirmação da deficiência da mesma. Por outro lado, por muitos anos foi consenso a utilização de vitamina $D$ no controle indireto dos níveis de PTH na condição de DMO-DRC.

O estudo de Hamdy NA, et al. (1995) utilizou como tratamento para o DMO-DRC, o alfacalcidol. Segundo esses autores houve resolução de $42 \%$ do distúrbio em comparação com placebo. Contudo, o uso do alfacalcidol somente estabilizou os níveis de fosfatase alcalina e PTH, ao contrário dos pacientes que utilizaram placebo, que aumentaram em muito os níveis séricos dessas substâncias.

Nesse estudo, os participantes que usaram o alfacalcidol desenvolveram a hipercalcemia e, embora tenham melhorado o quadro com a redução da dose, foram excluídos antes do término dos dois anos do estudo para proteção da saúde devido a todos os riscos para DCV, já discutidos até aqui. Um dado importante é que nenhum dos dois grupos alterou a TFGe durante o período de dois anos. Considerando agora a possibilidade de baixa de calcidiol na vigência de DMO-DRC, Dogan E, et al. (2008) utilizaram em o colecalciferol em grupos de pacientes com DRC e níveis baixos de calcidiol para determinar o efeito da suplementação oral de vitamina $D$ no ajuste dos marcadores de doenças ósseas urêmicas. Com o uso do colecalciferol após um mês de tratamento houve aumento do calcidiol, e diminuição do PTH, porém, sem alteração significativa nos níveis de cálcio e fósforo. Provavelmente devido ao curto prazo do estudo, não foi pesquisada possíveis alterações da FR.

Os autores sugeriram que essa é uma opção segura para tratar DMO-DRC nas fases iniciais, acompanhando de forma muito rigorosa também os níveis de cálcio e fósforo. Essa afirmativa foi corroborada por Oksa A, et al. (2008) que utilizaram o colecalciferol para correção da deficiência de vitamina $D$, e os resultados mostraram que além da correção de aproximadamente $50 \%$ dos níveis de vitamina $D$, também reduziram PTH e os níveis séricos de cálcio e fósforo não alteraram. Como no estudo de Dogan $\mathrm{E}$, et al. (2008) não foi avaliada a progressão da FR. Ambos os estudos citam o uso do colecalciferol como seguro para controle, em especial, do nível de PTH.

Já com foco nos riscos da hiperfosfatemia, Block GA, et al. (2012) estudaram os aglutinantes de fosfato (acetato de cálcio, o carbonato de lantânio e o carbonato de sevelamer). Os participantes desse estudo foram 
randomizados em quatro grupos: pacientes que receberam placebo e três grupos que receberam os diferentes aglutinantes de fosfato. Neste estudo, o fósforo sérico diminuiu de uma média basal de 4,2 para 3,9 mg / dl nos tratados com os aglutinantes de fosfato e não alterou nos tratados com placebo.

Somente o carbonato de lantânio, dentre os aglutinantes de fosfato, quando comparado com placebo, apresentou resultado efetivo. No entanto, os resultados deste estudo apontaram que os aglutinantes de fosfato promoveram a progressão da calcificação da artéria coronária e da aorta abdominal.

Novamente baseados nos resultados desse estudo vale alertar que, embora se tenha tido um bom controle do DMO-DRC com os aglutinantes de fosfato, houve uma grave resposta no sentido de potencialização do risco de DCV.

Exatamente pensando em diminuir o risco de CV Toussaint ND, et al. (2010) utilizaram doses de $70 \mathrm{mg}$ de alendronato por dezoito meses e placebo. No desfecho primário, embora tenha tido CV em ambos os grupos, não houve diferença na progressão da mesma, sugerindo que o alendronato não protegeu esses pacientes.

Nos desfechos secundários, os resultados mostraram CV da artéria femoral, aumento da densidade mineral óssea da coluna lombar em ambos os grupos e, mais grave, uma piora na FR e nos marcadores séricos do metabolismo mineral com o uso do alendronato.

Não houve registros de avaliação se as últimas alterações ocorreram pela evolução espontânea da DRC ou devido ao uso do alendronato. De qualquer forma, fica o alerta, que em casos de pacientes com DRC, o alendronato não foi efetivo para controlar a CV.

Outro exemplo da ação indireta da Vitamina D nos níveis de PTH foi identificado no trabalho de Isakova T, et al. (2012). Neste estudo, utilizou-se o calcitriol para testar a hipótese de que conjuntamente com cálcio na dieta iria controlar a hipocalcemia e diminuir os níveis de PTH. Os resultados mostraram que os níveis de PTH diminuíram e quando combinado com cálcio na dieta, normalizaram-se as respostas pós-prandiais de calcemia e do PTH.

Uma informação importante que não foi relatada nesse estudo foram os níveis séricos prévios de vitamina D nos pacientes submetidos ao tratamento do DMO. Assim, é preciso ter em mente que, embora os resultados tenham sido satisfatórios no sentido de diminuir PTH e normalizar níveis séricos calcêmicos, foram feitos somente durante uma semana. Por isso, não se pode perder de vista a reflexão das consequências dessa abordagem em longo prazo, principalmente considerando os riscos de CV e instalação de DCV.

Russo D, et al. (2015) avaliaram uma estratégia simultânea de dieta restrita de fósforo e terapia com o carbonato de cálcio ou sevelamer para avaliar se esta combinação atenuaria a progressão da calcificação da artéria coronariana $(C A C)$.

Os resultados ao longo de trinta e seis meses mostraram uma redução significativa da mortalidade por todas as causas, com progressão de CAC ausente ou moderada, mas não acelerada. Os resultados sugeriram o sevelamer como uma opção segura para diminuição da mortalidade por todas as causas, mas sem efeito sobre o avanço da CV.

Diante de todos os resultados apresentados pelos estudos elencados, observa-se que, quaisquer que sejam as propostas de abordagem a esses pacientes, o acompanhamento rigoroso dos níveis séricos de cálcio, fósforo, vitamina D e PTH devem ser tomados como base para ajuste de doses e tratamento, inclusive podendo sugerir a suspensão do mesmo.

Por fim, salienta-se que embora os resultados dessa revisão sejam de extrema importância para orientação de tomada de decisão na prática clínica, é preciso registrar limitações. Entre elas destaca-se a escassez de artigos com essa temática. $\mathrm{E}$ ainda, a falta de padronização dos dados coletados nos estudos elencados de forma a inviabilizar a realização da metanálise. Mesmo assim, considerando a avaliação das possibilidades de viés e nível de evidência dos estudos, a maioria apresentou risco médio e baixo de viés e média a alta qualidade da evidência gerada, o que agrega robustez e confiança nos resultados dessa revisão 


\section{CONSIDERAÇÔES FINAIS}

Não houve consenso entre os autores quanto à terapêutica padrão ouro de excelência para abordagem aos pacientes com DMO-DRC em estágios precoces não dialíticos, de forma a restabelecer os níveis séricos adequados do PTH, cálcio e fósforo, minimizar o risco de doença cardiovascular e manter a FR. Os benefícios de cada intervenção foram pontuais e, por vezes, equivalentes aos prejuízos decorrentes do tratamento são eles: uso de calcitriol ou colecalciferol, tendo calcitriol resultado melhor quanto a correção da hipocalcemia, redução das fosfatases alcalinas séricas e do hormônio paratireóideo; alfacalcidol com controle da doença óssea em $42 \%$ dos pacientes; colecalciferol aumentou calcidiol sérico e diminuiu níveis séricos de paratormônio; colecalciferol por tempo prolongado com melhora na deficiência de vitamina $D$ e diminuição dos níveis séricos de paratormônio; alendronato por tempo prolongado, não alterou a progressão da calcificação vascular; carbonato de lantânio ou carbonato de sevelamer diminuíram o fósforo médio de urina e atenuaram a progressão do hiperparatireoidismo; calcitriol associado a intervenção dietética de cálcio, calcitriol isolado diminuiu os níveis PTHi em jejum e, quando combinado com cálcio na dieta, normalizou as respostas pós-prandiais de calcemia e paratireoide e carbonato de cálcio ou sevelamer associados a dieta restrita de fósforo. O sevelamer apresentou melhores resultados com redução significativa na mortalidade por todas as causas.

\section{REFERÊNCIAS}

1. ABDU A, et al. Prevalence and pattern of chronic kidney disease-mineral bone disorders among hemodialysis patients in kano, northwest nigeria. 1르n Afr Med. 2019; 18(4):191-95.

2. ADRAGÃO T, et al. A simple vascular calcification score predicts cardiovascular risk in hemodialysis patients. Nephrol Dial Transplant. 2004; 19(6):1480-8.

3. ALCADE PR, KIRSZTAJN GM. Gastos do Sistema Único de Saúde brasileiro com doença renal crônica. J. Bras. Nefrol.2018; 40(2).

4. BALSHEMA H, et al. GRADE guidelines: 3. Rating the quality of evidence. J Clin Epidemiol. 2011; 64:401-6.

5. BASTOS MG, KIRSZTAJN GM. Chronic kidney disease: importance of early diagnosis, immediate referral and structured interdisciplinary approach to improve outcomes in patients not yet on dialysis. J Bras Nefrol. 2011; 33(1):7487.

6. BLOCK GA, et al. Effects of phosphate binders in moderate CKD. J Am Soc Nephrol. 2012; 23(8):1407-15. 27.

7. BLOCK GA, et al. Mineral metabolism, mortality, and morbity in maintenance hemodialysis. J Am Soc Nephrol. 2004; 15(8):2208-18.

8. CHRISTIANSEN C, et al. Decreased renal function in association with administration of 1,25-dihydroxyvitamin D3 to patients with stable, advanced renal failure. Contrib Nephrol. 1980; 18:139-46.

9. CHRISTIANSEN C, et al. Deterioration of renal function during treatment of chronic renal failure with 1,25dihydroxycholecalciferol. Lancet.1978; 30(2):700-3.

10. DOGAN E, et al. Effect of depot oral cholecalciferol treatment on secondary hyperparathyroidism in stage 3 and stage 4 chronic kidney diseases patients. Ren Fail. 2008; 30(4):407-10.

11. FILHO AJI, MELAMED ML. Vitamina D e doença renal. O que nós sabemos e o que nós não sabemos. J. Bras. Nefrol. 2013; 35(4).

12. FUJII H. Chronic kidney disease - CKD-MBD and cardiovascular disease. Clin Calcium. 2019; 29(2):179-184.

13. GUÉRIN AP, et al. Arterial stiffening and vascular calcifications in end-stage renal disease. Nephrol Dial Transplant. 2000; 15(7):1014-21.

14. HAMDY NA, et al. Effect of alfacalcidol on natural course of renal bone disease in mild to moderate renal failure. BMJ. 1995; 310(6976):358-63.

15. ISAKOVA T, et al. Daily variability in mineral metabolites in CKD and effects of dietary calcium and calcitriol. Clin $\mathrm{J}$ Am Soc Nephrol. 2012; 7(5):820-8.

16. JIN JJ, et al. Prevalence of Chronic Kidney Disease-Mineral Bone Disorder in Hemodialysis Patients in Hebei, China. Chin Med J. 2018; 131(22):2749-51.

17. KANBAY M, et al. Association of serum calcitonin with coronary artery disease in individuals with and without chronic kidney disease. Int Urol Nephrol. 2013; 44(4):1169-75.

18. MINISTÉRIO DA SAÚDE. Portaria no 801. Dispõe sobre a aprovação do Protocolo Clínico e Diretrizes Terapêuticas TGP do Distúrbio Mineral Ósseo na Doença Renal Crônica. Brasília: Ministério da Saúde, 2017.

19. MOHER D, et al. PRISMA Group.Preferredreportingitems for systematicreviewsand meta-analyses: the PRISMA statement. PLoS Med. 2009; 21;6(7).

20. NATIONAL KIDNEY FOUNDATION. Clinical practice guideline for the evaluation and management of chronic kidney disease. Kidney Inter Suppl. 2012; 3(1):1-150.

21. NATIONAL KIDNEY FOUNDATION. Clinical Practice Guideline Update or the Diagnosis, Evaluation, Prevention, and Treatment of Chronic Kidney Disease-Mineral and Bone Disorder (CKD-MBD). Kidney Int. 2017; 7(1):1-59. 
22. OKSA A, et al. Effects of long-term cholecalciferol supplementation on mineral metabolism and calciotropic hormones in chronic kidney disease. Kidney Blood Press Res. 2008; 31(5):322-9.

23. ROETKER NS, et al. Adherence to Kidney Disease: Improving Global Outcomes Mineral and Bone Guidelines for Monitoring Biochemical Parameters. Am J Nephrol 2019; 49:(3)225-232.

24. RUSSO D, et al. Effects of phosphorus-restricted diet and phosphate-binding therapy on outcomes in patients with chronic kidney disease. J Nephrol. 2015; 28(1):73-80.

25. SPASOVSKI G, et al. Current status of transplantation and organ donation in the Balkans-could it be improved through the South-eastern European health Network (SEEHN) initiative? Nephrol Dial Transplant. 2012; 27(4):1319-23.

26. STENGEL B, et al. Epidemiology and prognostic significance of chronic kidney disease in the elderly-the Three-City prospective cohort study. Nephrol Dial Transplant. 2011; 26(10):3286-95.

27. SOCIEDADE BRASILEIRA DE NEFROLOGIA. IN: Censos e Registros de 2019. Brasil. Disponível em: http://www.sbn.org.br. Acesso em: 24 mai. 2020.

28. TOUSSAINT ND, et al. Effect of alendronate on vascular calcification in CKD stages 3 and 4: a pilot randomized controlled trial. Am J Kidney Dis. 2010; 56(1):57-68.

29. VALENTE LM, et al. Diagnóstico e intervenções para retardar a progressão da doença renal crônica em hipertensos e diabéticos. Bvs. Universidade Federal do Maranhão, Maranhão, 2015.

30. ZUMNAN MG, et al. $2^{\underline{a}}$ Secondary hyperparathyroidism among Nigerians with chronic kidney disease Nephrology Division. Afr Health Sci. 2018; 18(2)446-57. 